\title{
Frame vs. Trajectory Analyses of Pedestrian Dynamics Asymmetries in a Staircase Landing
}

\author{
Alessandro Corbetta ${ }^{1}$. Chung-min Lee ${ }^{2}$. Adrian Muntean ${ }^{3}$. \\ Federico Toschi ${ }^{4}$ \\ ${ }^{1}$ Eindhoven University of Technology, Eindhoven, The Netherlands, \\ E-mail: a.corbetta@tue.nl \\ 2 California State University Long Beach, Long Beach, CA, USA, \\ E-mail: chung-min.lee@csulb.edu \\ ${ }^{3}$ Karlstad University, Karlstad, Sweden, \\ E-mail: adrian.muntean@kau.se \\ 4 Eindhoven University of Technology, Eindhoven, The Netherlands \& CNR-IAC, Rome, Italy, \\ E-mail: f.toschi@tue.nl
}

Received: 12 June 2016 / Accepted: 3 February 2017

DOI: $10.17815 / C D .2017 .10$

\begin{abstract}
Real-life, out-of-laboratory, measurements of pedestrian walking dynamics allow extensive and fully-resolved statistical analyses. However, data acquisition in real-life is subjected to the randomness and heterogeneity that characterizes crowd flows over time. In a typical real-life location, disparate flow conditions follow one another in random order: for instance, a low density pedestrian co-flow dynamics may suddenly turn into a high density counter-flow scenario and then back again. Isolating occurrences of similar flow conditions within the acquired data is a paramount first step in the analyses in order to avoid spurious statistics and to enable qualitative comparisons.

In this paper we extend our previous investigation on the asymmetric pedestrian dynamics on a staircase landing, where we collected a large statistical database of measurements from ad hoc continuous recordings [1]. This contribution has a two-fold aim: first, method-wise, we discuss an analysis workflow to consider large-scale experimental measurements, suggesting two querying approaches to automatically extract occurrences of similar flow scenarios out of datasets. These pursue aggregation of similar scenarios on either a frame or a trajectory basis. Second, we employ these two different perspectives to further explore asymmetries in the pedestrian dynamics in our measurement site. We report cross-comparisons of statistics of pedestrian positions, velocities and accelerations
\end{abstract}


vs. flow conditions as well as vs. querying approach.

Keywords Pedestrian dynamics $\cdot$ high statistics measurements $\cdot$ statistical mechanics · data analysis

\section{Introduction}

Experimental investigations of pedestrian dynamics for behavioral insights or predictive model validation saw a rapid proliferation over the last years [2-6]. Fine-scale data collections via pedestrian tracking have been growing in complexity and acquisition scales [7-9], both in $[10,11]$ and out $[6,12,13]$ of controlled laboratory environments.

Laboratory experiments enable detailed parametric studies of the crowd flow (see, e.g., [14]), and may employ visual markers, such as colored shirts and/or helmets, to enhance automatic pedestrian detection and tracking [9]. Real-life condition measurements, recently tackled via, e.g., wireless sensors [15] or, as here, via 3D sensors [10, 12, 13], likely eliminate potential behavioral biases introduced by a laboratory environment, such as the awareness of being part of a scientific experiment. Furthermore, measurements in real-life enable and are necessary if one aims at resolved statistical descriptions of physical observables (e.g., positions, velocities, accelerations) or to quantify related rare events $[16,17]$. In this case, large amount of data are required, and they can be obtained via accumulating measurements with continuous and long-time ranged experimental campaigns $[8,12]$. From the technical point of view, real-life measurements present the challenge for computer vision to identify pedestrians automatically (see e.g. [18] for an overview on color image based pedestrian detection techniques, or the previous references for the usage of 3D sensors).

As a significant difference from laboratory settings, where experiments are design defining, geometry [7,10], but also age [19], competitiveness [20] of participants and so on, real-life measurements are subjected to the natural uncontrollability and unpredictability of pedestrian flows. In fact, real-life measurements unavoidably include heterogeneous scenarios (cf. sketch in Fig. 1): for instance, in a train station during the rush hour, a low density pedestrian flow can suddenly turn into a dense crowd [16,21]. Similarly, individuals walking undisturbed can alternate with group dynamics [22]. Real-life crowd data have been often analyzed regardless of the mixed flow scenarios [8], e.g. to evaluate the flow statistics globally or to report time-histories. In many occasions, nonetheless, when aiming at understanding the dynamics, a (possibly implicit) preliminary data screening is applied to ensure the aggregation and analysis just of occurrences of similar (homogeneous) flow conditions. In fact, we expect that pedestrians walking isolated from peers will exhibit a different dynamics than pedestrians walking in groups [23]. Thus, drawing statistics of data inclusive of these two heterogeneous flow conditions unavoidably yields ambiguous statistics. In general, the classification of flow conditions poses a challenge when working with vast datasets. Conventionally, manual data selection and annotation has been exclusively employed, e.g. to select groups in [23], to classify walking patterns in [24], or to isolate people waiting in [25]. Although manual annotation is certainly a 
possibility for processing data acquired in laboratories or in real-life at small scales, it may become prohibitively time consuming when dealing with extensive real-life recordings. Performing these classification operations automatically for pedestrian dynamics analysis is, to the best of our knowledge, an open problem.

The first goal of this work is to discuss the analysis workflow when dealing with large scale, widely heterogeneous, measurement ensembles and to suggest strategies for automatized selection and aggregation (queries according to the established database terminology) of homogeneous sub-sets of data.

This work is developed in reference to specific extensive measurements collected by us in a year-long real-life measurement campaign at Eindhoven University of Technology, the Netherlands [12]. During this campaign we recorded on a 24/7 schedule pedestrian trajectories in a landing (intermediate planar area between flights of stairs) with corridorlike geometry (further details in Sec. 3). Staircases landing are relevant scenarios for crowd dynamics, yet just partially explored, mainly in the context of evacuation dynamics (see, e.g. [26-29] and references therein). Passage by landings is in fact obliged in case of evacuations via stairs. Ordinary pedestrian dynamics on a landing is heterogeneous, as it includes scenarios such as uni- or bi-directional flows with one or several pedestrians. Furthermore, it is naturally asymmetric due both to cultural preferences for the walking side [30] and to the fact that individuals are either ascending or descending the neighboring stair flights (for a reference of pedestrian dynamics on stairs, see, e.g. [26,31]). From our preliminary overview of the traffic in [1], we expect the dynamics in the corridor to be driven by two elements: the number of pedestrians in the landing (taken as a surrogate of the density) and their walking directions.

The second aim of this work is to evaluate the asymmetric pedestrian dynamics in a staircase landing, cross-comparing different flow regimes in terms of statistics of pedestrian position, velocity and acceleration. Relevantly, the two considered driving elements are insufficient in defining an aggregation strategy for homogeneous flow conditions. With more clarification provided in Sec. 2, these two driving parameters can be used to aggregate data at the time frame level (for a frame-based query) or at the trajectory level (for a trajectory-based query). While the first approach appears to be a natural choice privileging simplicity, the second deems that long-range mutual interactions and memory effects are expected to influence the dynamics beyond a single frame, steering entire trajectories. Hence, we also employ selected flow conditions to compare the two querying approaches.

The content of the paper is organized as follows: in Sec. 2 we discuss the experimental workflow when considering large scale experimental measurements and formally introduce the concepts of frame-based and trajectory-based queries for pedestrian dynamics datasets. The data analyses of our dataset are the subject of Sec. 3. The opportunity to compare the querying strategies at work is also taken within the section. A discussion in Sec. 4 closes the paper. 


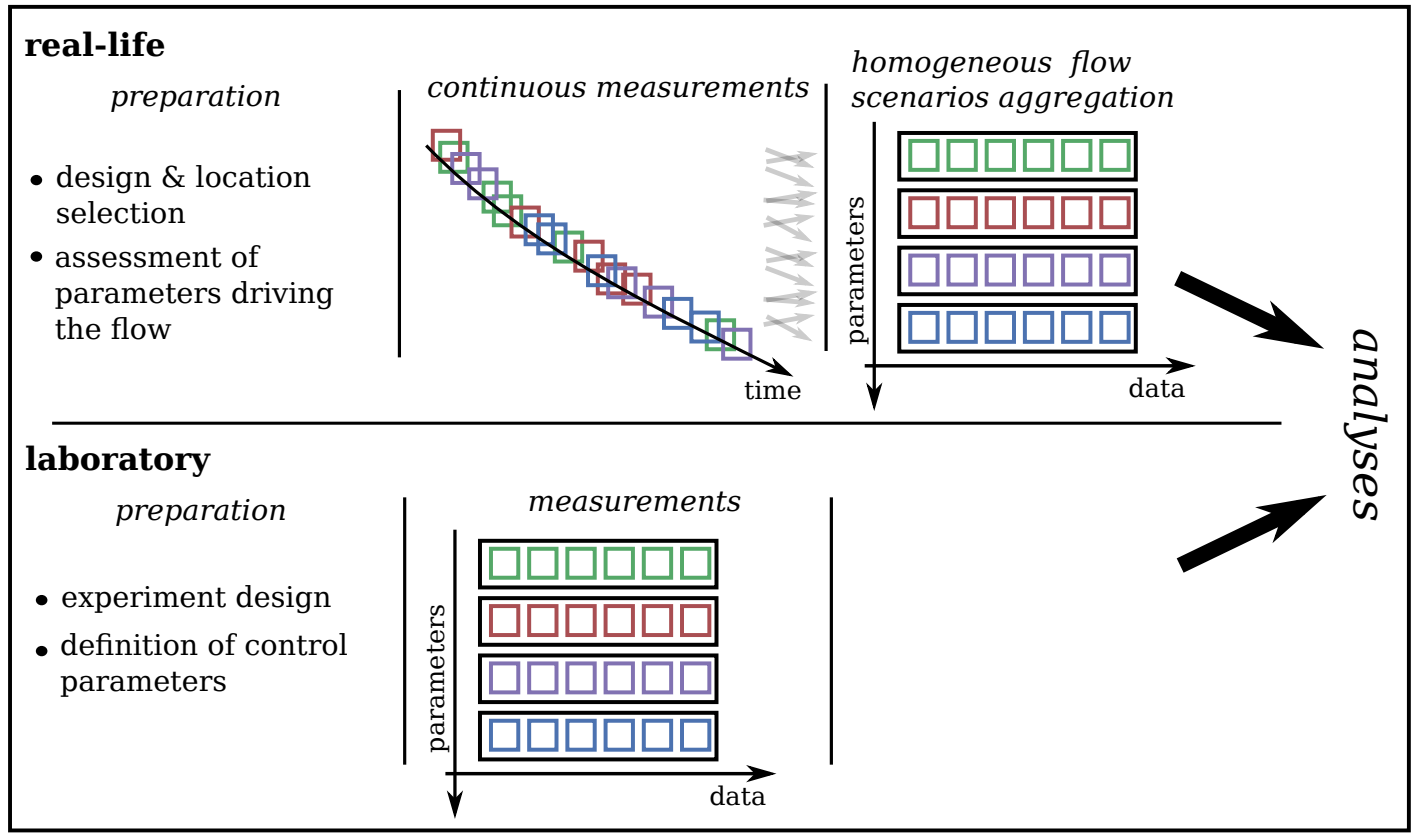

Figure 1 Experimental workflow simplified schematics in cases of real-life continuous measurements (top) and laboratory-like measurements (bottom). In both cases, one aims at (statistical) analyses and cross-comparisons of measurements at different flow conditions. In a laboratory environment the control parameters of an experiment (e.g. crowd density, crowd composition, geometric properties of the environment, etc.) can be set a priori, before recording and analyzing the data. In real-life measurements, we continuously record alternating heterogeneous dynamics without dictating the flow conditions (cf. "continuous measurements" panel vs. Fig. 4(b1-d1)), which are in turn ruled by unbiased individual motivations. We represent schematically measurements with squares, whose color is associated to the control parameters. In case of real-life measurements an intermediate step of identification and aggregation of instances of similar (homogeneous) flow conditions (represented with gray arrows) is thus necessary before the data analyses. In Sec. 2 we discuss automatic procedure for such a step.

\section{Aggregation of homogeneous measurements: frame- and trajectory-based queries}

In this section we provide definitions and examples for frame-based and trajectory-based data queries. First, the aim of a query is to obtain a sub-set of experimental data satisfying given conditions. The measurement data come in the form of frames which are records of pedestrian positions over time. Considering experiments that took place in real-life settings, measurements can possibly span over long time periods with very different flow conditions. To systematically investigate the dynamics presented in the data, one needs to isolate occurrences of similar dynamics according to flow conditions before applying statistical analyses. The criteria of query should be pedestrian flow features that are expected to play significant roles in the dynamics. For instance, for the experimental scenario considered in Fig. 4, the number of pedestrians present in the monitored area and their walking directions are expected to be the major drivers of the dynamics [1]. Notably, conceptually similar queries can be performed at the level of frames or trajectories. 
As discussed in the example below and in the forthcoming analyses, such queries might yield considerably different datasets (hence physical observations). Thus the precise definitions of frame- and trajectory-based queries and comparisons are deemed relevant. In particular, a frame-based query collects measurements when given conditions are met on a frame-by-frame basis. On the contrary, a trajectory-based query selects data specifying conditions over entire trajectories, thus going beyond what occurs in single frames. Therefore, data are retained only if the given condition is verified through the whole trajectory.

In the remaining part of the section we delineate the previous general considerations to experimental scenarios like narrow corridors, as considered in Fig. 4. In these situations pedestrian mainly walk in the longitudinal direction joining the two ends of the walkway. Consistently with the reference introduced in Fig. 4(b2-d2), we identify these two possible walking directions as left-to-right (2R), i.e., walking from the left end to the right end of the walkway, and as right-to-left (2L), which is the opposite case (in the following, for the sake of brevity, the short notation $2 \mathrm{~L} / 2 \mathrm{R}$ or plain arrows are used).

Considering the number of involved pedestrians per each of the two walking direction as dominant factor of the dynamics, we list few examples of queries. These also provide the case studies for the forthcoming sections of the paper:

(Q1) considering all the frames in which one pedestrian appears alone in our corridor with a given walking direction specifies a frame-based query;

(Q2) generalizing (Q1), we can aggregate all time frames in which a given number of pedestrians with specified walking directions are in the facility. This is another frame-based query.

(Q3) case (Q1) query yields frames showing only one pedestrian. Considering the pedestrian $(\mathrm{P})$ within a given frame, there are only two, mutually exclusive, cases: (a) $\mathrm{P}$ remains alone in the scene throughout his or her entire trajectory, thus all frames constituting the trajectory are selected by the query; (b) in a frame that comes earlier or later in time, $\mathrm{P}$ shares the measurement region with one or more other pedestrians. We name a pedestrian in case (a) undisturbed, as he or she does not have any interaction while walking within our measurement region. Isolating all the trajectories by undisturbed pedestrians (with an assigned walking direction) defines a trajectory-based query;

(Q4) the simplest avoidance scenario involves exactly two pedestrians (e.g. P3 and P4 in Fig. 2) walking in opposite directions. To ensure that the mutual presence is the only element influencing the pedestrian, we require that no third pedestrian is present except for P3 and/or P4 during their time in the landing. Once more, this is a trajectory-based query as it concerns a property of trajectories of $\mathrm{P} 3$ and $\mathrm{P} 4$ as a whole, i.e., no third pedestrian shares any frame in trajectories of P3 and P4.

In the following, when convenient, we will refer to trajectory-based queries and to framebased queries respectively through the abbreviations TBQ and FBQ. 

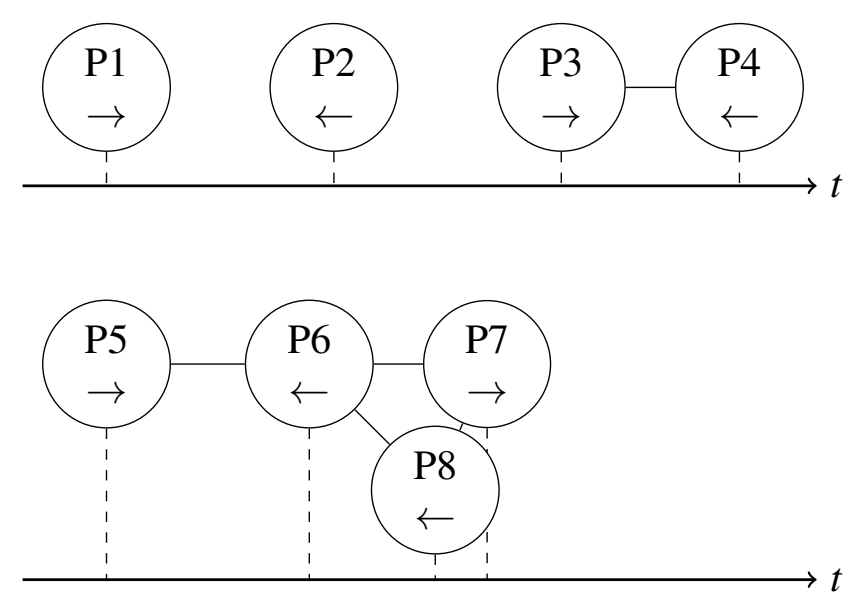

Figure 2 Flow conditions in their graph representations. We associate each pedestrian trajectory to a graph node, such node carries an annotation of the walking direction (right-to-left/left-to-right, cf. arrow $\leftarrow / \rightarrow$ ). We connect with an edge all those pedestrian/nodes that appear together in at least one time instant (frame) and define an interaction network. The entrance time of each pedestrian assigns an ordering for the nodes, and the time axis indicates such temporal order. In the upper panel, P1 identifies a pedestrian walking undisturbed (i.e. appearing alone along the entire trajectory) left-to-right (2R). P1 is in fact represented by a singleton node with no edges. Later in time, P2 crosses the facility walking undisturbed too, although going right-to-left (2L). Finally, P3 and P4 cross the facility. They have opposite walking direction and P3 enters first. They appear together in at least one time instant (frame), thus we assume that they interacted. Beside themselves, they do not appear with any other individual. Therefore P3 - P4 constitutes an stand-alone interaction network. Cases P1, P2, P3, P4 are considered in Sec. 3.3. A more complex interaction network is reported in the lower panel for pedestrians P5 - P8. P5 enters first, before leaving he/she shares the landing for at least one time frame with P6. Afterwards, P6 appears together with both P7 and P8. Trajectory-based queries (TBQ) select data from a dataset for interaction networks with specified properties on size and walking directions.

\subsection{Trajectory-based queries: interpretation and evaluation}

As described in the cases $(Q 3)$ and $(Q 4)$ above, trajectory-based queries consider one pedestrian's relations with one another or the lack thereof, where pedestrians are considered in interaction in case appeared together within our observation window. Therefore a relationship structure among pedestrians is necessary. We use undirected graphs consisting of nodes and edges between nodes to represent pedestrian relations (we refer, e.g., to [32] for an introduction on graphs). To construct the graph, first we associate each distinct pedestrian to a graph node, including the pedestrian's direction. Then we connect an edge between two nodes in case the two pedestrians appear together at least in one time frame. Analyzing the connected components of such a graph, i.e. those subgraph in which each node is connected to all others via a path constituted of one or more edges [32], that we name interaction networks, we can extract from our dataset measurements featuring homogeneous flow conditions with respect to trajectories.

For instance, pedestrians walking undisturbed are identified by interaction networks with just one node (e.g. P1 and P2 in Fig. 2). Interaction networks with two nodes identify scenarios involving exactly two pedestrians (cf. P3 -P4 in Fig. 2). In case opposite 


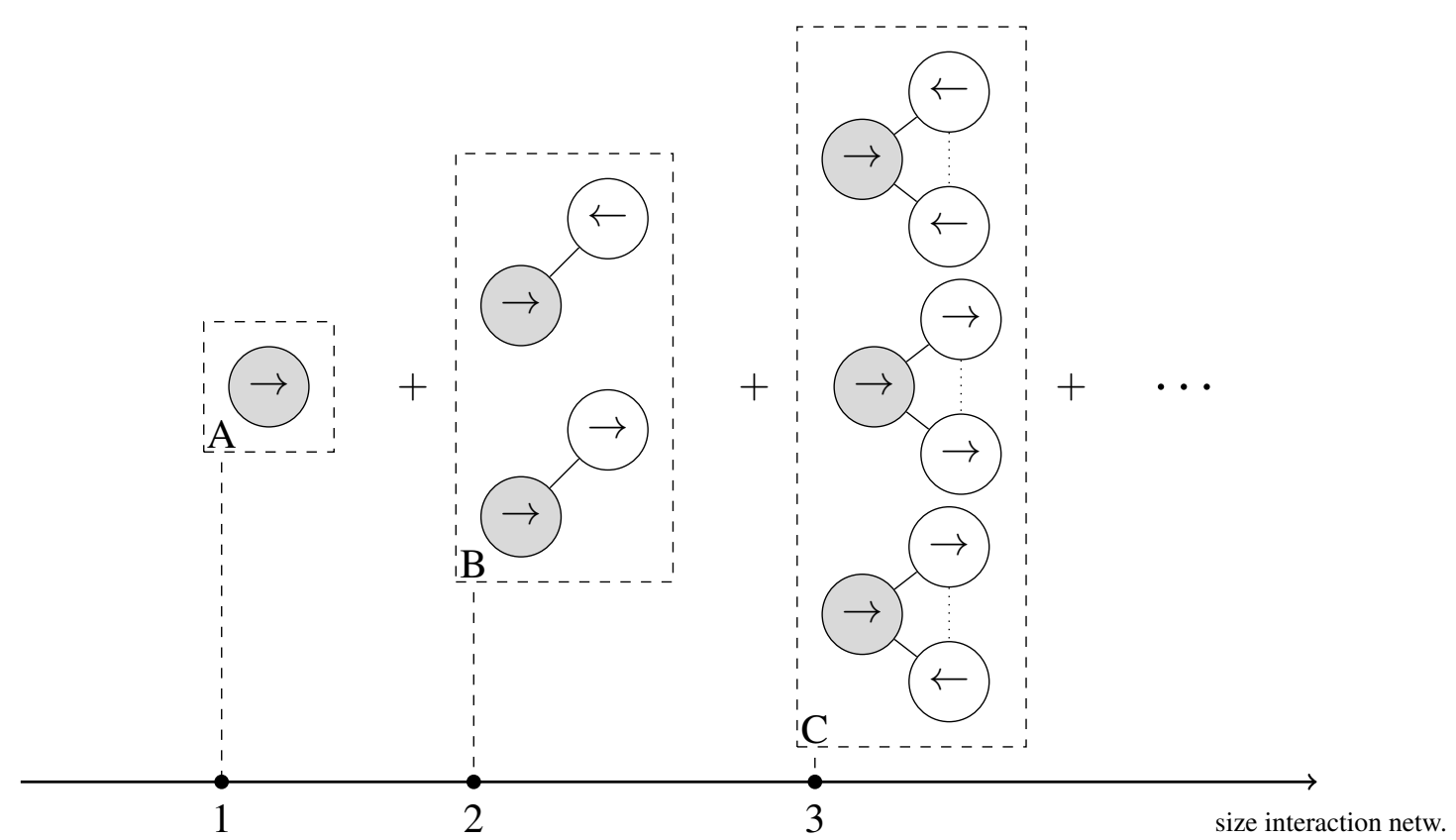

Figure 3 Ideal break up of a frame-based query (FBQ) for single pedestrians walking left-to-right (case (Q1) in Sec. 2) considering the different heterogeneous scenarios that make up the query results. We employ the graph description from Sec. 2.1 to group the contributing scenarios, and we order them by the size of the interaction network (horizontal axis). Identifying the trajectory contributing data to the FBQ with a grey node, we have: (A) contributions from pedestrians actually walking left-to-right undisturbed by other pedestrians. Such data would be selected by the equivalent TBQ (case (Q3) in Sec. 2); (B) contributions from pedestrians that also appear (in frames discarded by the current FBQ) with a second individual. The two pedestrians might walk in counter-flow (top case, as P3-P4 in Fig. 2) or in co-flow (bottom case); (C) contributions from pedestrians that also appear (only in frames discarded by the current FBQ) with two further pedestrians. These two pedestrians may themselves or may not appear at the same time. Hence an edge shall or shall not connect them (thus the dotted edge). The sequence of combinations can clearly grow indefinitely, within the physical limits of the observed area.

walking directions are further associated to these, avoidance scenarios are identified (as considered in Sec. 3.3)

Notably, this graph based selection comes at low computational costs as: (i) one pass of the dataset is sufficient to build the graph; (ii) querying for connected components is a light operation on modern graph software libraries such as [33]. We can use this graph representation to interpret further the difference between frame-based and trajectory-based queries. As examples, we consider the queries $(Q 1)$ and $(Q 3)$ in Sec. 2. Following (i) we isolate all the time frames in which one pedestrian walking in a given direction (e.g. from the left side of the corridor to the right side) is observed. Measurements possibly from several trajectory fragments of a pedestrian remain thus aggregated. In Fig. 3 we report a trajectory-based classification of these trajectory fragments. There, the number of nodes of the connected components to which each trajectory fragment belongs gives the sorting criterion. Hence, the query $(Q 1)$ includes the entire selection given by $(Q 3)$ (connected components with just one node) plus measurements from pedestrians that in previous or 
future time frames will appear with one, two or more other individuals. The following observations are due:

- frame-based queries (e.g., (Q1)-(Q2) in Sec. 2) aggregate conditions having similar load and/or analogous usage patterns of the corridor;

- conversely, trajectory-based queries (e.g., (Q3)-(Q4) in Sec. 2) identify specific physical scenarios focusing on the involved pedestrians (cf. cases P1 - P4 in Fig. 2). In general these scenarios appear as ideal references in social-force-like modeling perspectives [34], where the pedestrian motion is a sum of a desired component in absence of other individuals (as for P1 or P2), plus additive term considering pairwise interactions (as for P3-P4). See Sec. 3.3 for further modeling considerations.

- The expansion in Fig. 3 grows with a super-exponential [35] number of different graph configurations as the number of considered pedestrians increases. This means that a graph based description may become impractical to analyze dense crowds, and frame-based queries may remain the only option. Nevertheless, in those conditions, it seems reasonable that the dynamics is driven by density effects rather than the exact sequence of co-presences in the scene (graph edges). In other words, in case of large and highly connected graphs, we expect strong similarities in the dynamics, independently on the exact structure of the connections.

\section{Asymmetric dynamics in a staircase landing}

We employ frame- and trajectory-based queries to select and analyze data from our large scale real-life measurements of pedestrian dynamics in a corridor-shaped landing. For the sake of completeness we report here a primer of the measurement campaign and we refer interested readers to $[1,12]$ for a more detailed overview of the traffic and to $[16,36]$ for the techniques employed.

In the one year starting from October 2013 we recorded via an overhead Microsoft Kinect $^{\mathrm{TM}}$ 3D-range sensor [37] all pedestrians walking in a landing within the Metaforum building at Eindhoven University of Technology. The landing connects two staircases in the configuration presented in Fig. 4(a), where individuals ascend in a clockwise direction from the ground floor to the first floor of the building. The landing is $5.2 \mathrm{~m}$ long and $1.2 \mathrm{~m}$ wide, and the steps have the same width. Individuals at the ground floor reach the landing after 18 steps, then they climb 4 further steps arriving at the first floor. Recordings went on a 24/7 basis and include data from 108 working days. With ad hoc processing techniques of the Kinect ${ }^{\mathrm{TM}}$ depth cloud and particle-in-flow type tracking $[38,39]$, we collected $c a$. 230,000 time-resolved high-resolution trajectories.

Trajectories span diverse flow scenarios, ranging from pedestrians walking alone, undisturbed, to multiple pedestrians sharing the same walking direction, i.e. co-flowing, to clogged counter-flows, in which both motion directions are present. The statistics analysis from these flow scenarios is organized in the next subsection as follows: Sec. 3.1 

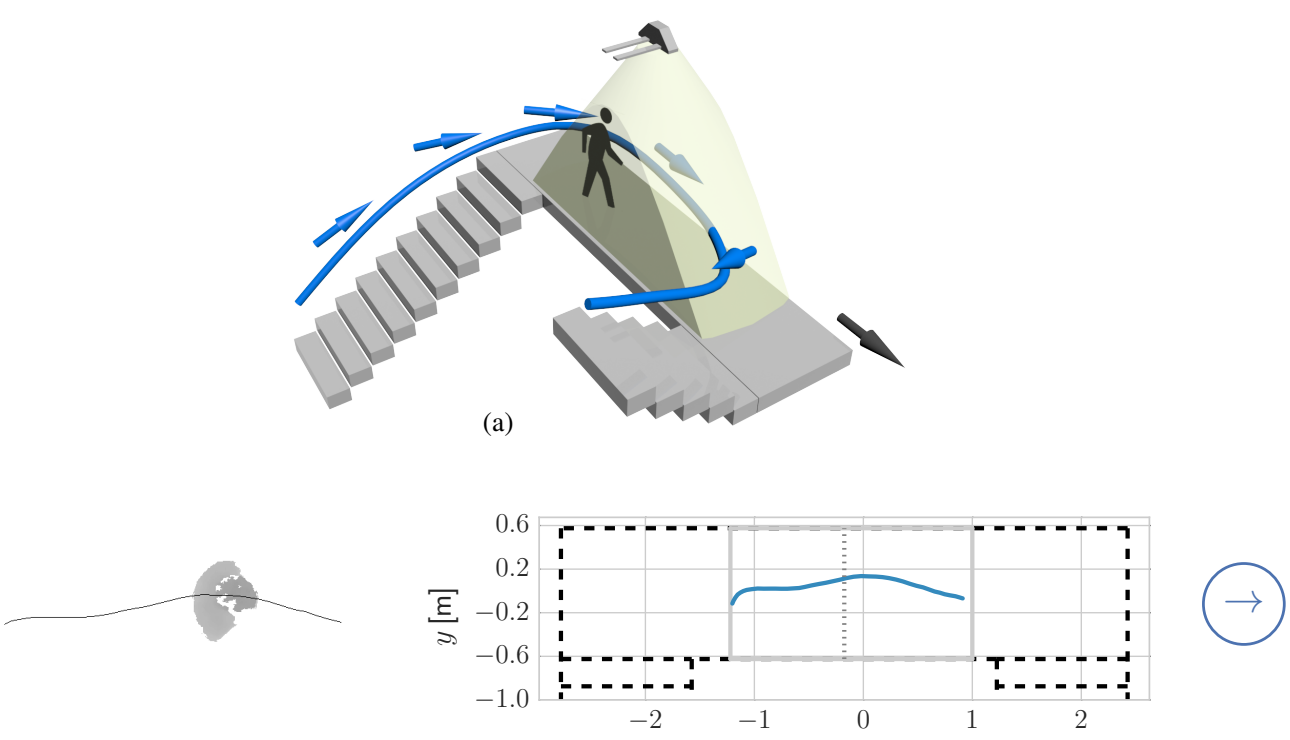

(b1)

(b2)

$x$ [m]

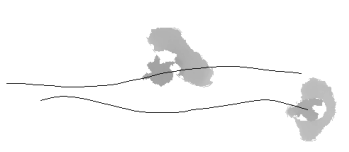

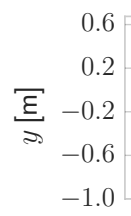

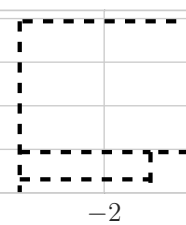

$$
\begin{array}{r} 
\\
\vec{E}-6 \\
0.2 \\
\hline \overrightarrow{\underline{E}}-0.2 \\
-0.6 \\
-1.0
\end{array}
$$

(d2) (c2)

$$
x[\mathrm{~m}]
$$

(c3)

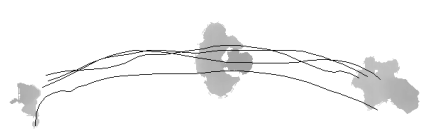

(d1)

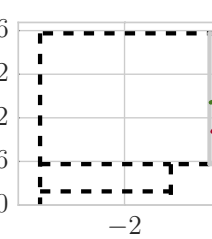

)

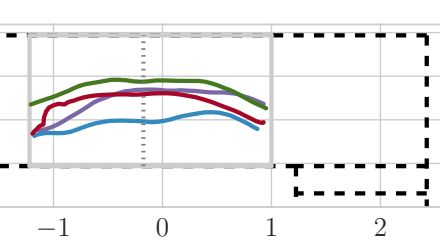

$x[\mathrm{~m}]$
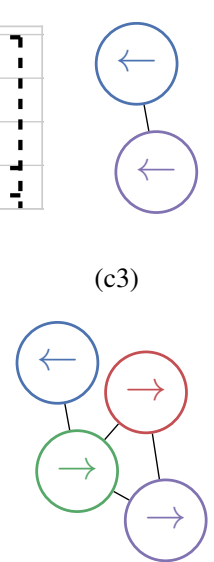

(d3)

Figure 4 (a) 3D sketch of the recorded landing and Kinect ${ }^{\mathrm{TM}}$ recording area. An ideal sample trajectory (analogous to panel b) from the left end to the right end of the facility (left-to-right, 2R) is reported. The whole area is surrounded by walls, here removed for readability. (b1-d1) Depth frames acquired in the landing by the Kinect ${ }^{\mathrm{TM}}$ sensor (background removed). The measured trajectories are superimposed as solid lines. (b2-d2) Measured trajectories reported within a sketched top view of the landing. The top view reports also the $(x, y)$ reference system considered. (b3-d3) interaction network of each condition. (b) One pedestrian walking the landing undisturbed in left-to-right direction $(2 \mathrm{R}, \rightarrow)$. (c) Two pedestrians co-flowing in right-to-left direction $(2 \mathrm{~L}, \leftarrow)$. (d) A frame containing three pedestrians is reported including all the four trajectories of their interaction network (cf. Fig. 2 P5 - P8).

provides an overview of the scenarios via frame-based queries; focusing on diluted scenarios involving one or two pedestrians, Sec. 3.2 compares the dynamics as observable from frame- and trajectory-based queries discussing the differences. Finally, a thorough analysis up to the pedestrian "social interaction force" of the asymmetric dynamics of 


\begin{tabular}{r|rrrr}
\hline$\times \rightarrow$ & $\times 0$ & $\times 1$ & $\times 2$ & $\times 3$ \\
\hline$\times 0$ & & & & \\
\hline$\times 1$ & $1,631,573$ & 127,362 & 16,423 & 370 \\
$\times 2$ & 290,263 & 24,697 & 3,443 & 82 \\
$\times 3$ & 11,993 & 897 & 95 & \\
\hline
\end{tabular}

Table 1 Total number of frames selected via the frame-based queries in Fig. 5. Data are arranged in the same double entry fashion as Fig. 5: the number of pedestrians walking in direction left-toright $(\rightarrow)$ increases with the columns, while the number of pedestrians walking right-to-left $(\leftarrow)$ increases with the rows.

diluted pedestrian scenarios in the landing is carried out in Sec. 3.3.

\subsection{Frame-based overview of the dynamics}

The U-shape of the landing influences the dynamics of pedestrians that follow curved trajectories to reach the staircase at the opposite end of the walkway (cf. trajectories samples in Fig. 4). While passing by the landing, pedestrians are furthermore moving between two different floors of the building. According to the direction convection introduced in Sec. 2, the left-to-right (i.e. 2R) walking direction is to ascend, while the right-to-left (2L) direction is to descend. Shape and function differences among walking directions allow the emergence of asymmetries in the dynamics of and within the different flow conditions (undisturbed pedestrian vs. multiple pedestrians vs. direction. See also our previous work [1]).

First we give an overview of the dynamics by means of an extensive frame-based querying over all the observed combinations of number of pedestrians and of walking directions (cf. Sec. 2.1). Curved pedestrian trajectories fall preferentially in narrow curved bands, that we name preferred position bands. We evaluate such bands by (a) binning the local pedestrian position data $(x, y)$ according to the span-wise coordinate $x$; (b) evaluating statistics (e.g. percentiles in distributions) on the transversal coordinate $y$. In Fig. 5, the bands range from the $15^{\text {th }}$ to $85^{\text {th }}$ percentiles of the local $y$ coordinate value (cf. App. A for technical details).

We observe that pedestrians walk through the landing maintaining the relative right, this occurs independently on the specific flow configuration. As the number of co-flowing pedestrians increases, the width of the preferred position band increases, and, as the number of counter-flowing pedestrians increases, the preferred position band becomes narrower. As intuition suggests, the widths of preferred position bands in the counter-flow situation roughly follows the ratio between the numbers of pedestrians in both directions. Note that the cases with highest load ( 5 pedestrians in total) are rarely measured, thus the poorer statistics and the decreased smoothness of the positions bands in Fig. 5. For an overview of the average walking velocities across these frame-based queries we refer the readers to Sect. 3 in [1]. 

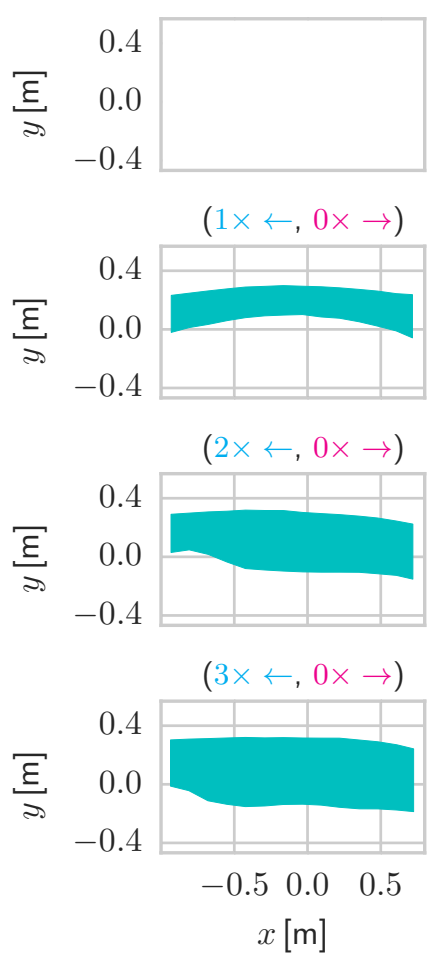
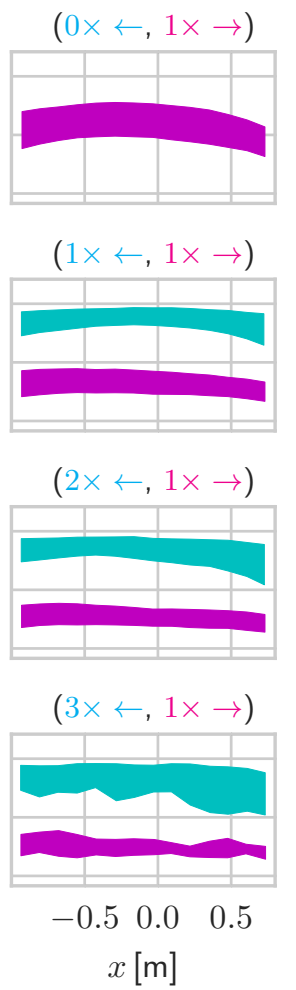
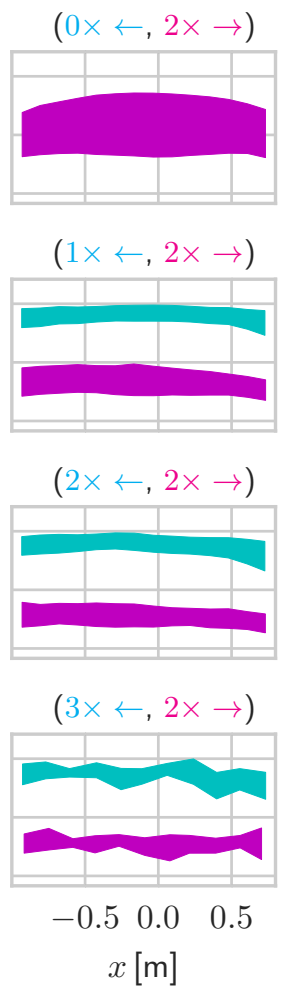
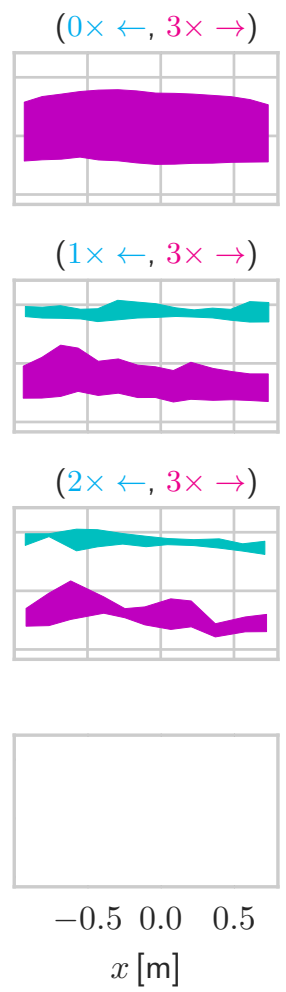

Figure 5 Frame-based analysis of preferred position bands in dependence on the number of pedestrians per walking direction. Subplots are arranged in a double-entry fashion, with each plot reporting on a different frame-based query. Each query, written schematically as subplot title, considers the number of pedestrians walking left-to-right (increasing from 0 to 3 moving rightwards through the plots), or right-to-left (increasing from 0 to 3 moving downwards through the plots). For instance, the plot $(2 \times \leftarrow, 1 \times \rightarrow)$ reports on the condition where two pedestrians walk rightto-left and one walks left-to-right ( 3 in total). The preferred position bands (i.e. the bands between the 15-th and 85-th percentiles of the distribution of the $y$ position component at each $x$ location, cf. App. A for technical details) are reported in cyan and in magenta, respectively for pedestrians walking right-to-left and for pedestrians walking left-to-right. All the data from pedestrians walking right-to-left (in this case 2 measurements per frame) contribute to the cyan band, and analogously for the magenta band (in this case 1 measurement per frame). Pedestrians conforms to the driving side preference by walking on the relative right side of the corridor, at least whenever a counter-flow occurs. An increase of co-flowing pedestrians results in an expanded preferred position band in the transversal direction $y$. Contrarily, an increase of counterflowing pedestrians constricts the width of the preferred position band. The total number of frames selected by each query is reported in Tab. 1 .

\subsection{Frame- vs. trajectory-based queries of diluted flows}

In Sec. 3.1 we aggregated our measurements via frame-based queries showing position preferences based on the walking direction. As commented in Sec. 2.1, frame-based queries exchange querying simplicity for physical clarity. When we consider trafficked dynamics involving many pedestrians, because of the combinatorial explosion of the trajectory graph configurations (cf. Fig. 3), frame-based queries are likely the only option. 
Nevertheless, these queries mix data from heterogeneous physical scenarios. In this section we compare the data selected by FBQ and TBQ when considering low density, diluted, flow conditions. Specifically, we consider and compare

(F1) the single pedestrian dynamics data selected by either queries, i.e., respectively, cases $(Q 1)$ and (Q3) in Sec. 2;

(F2) the pair counter-flow dynamics data selected by either queries. A FBQ for this scenario would select all the frames including exactly two pedestrians walking in opposite direction (as in Fig. 5, subplot " $(1 \times \leftarrow, 1 \times \rightarrow$ )"). Instead, a TBQ would select interaction networks of size 2 (cf. Sec. 2.1) in which both walking directions are present (i.e. P3-P4 in Fig. 2).

The following analyses include both bands of preferred position, as in Sec. 3.1, as well as walking velocity fields.

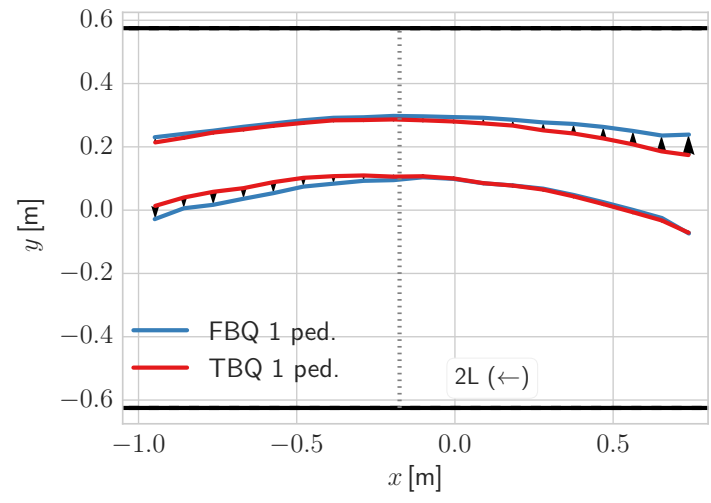

(a)

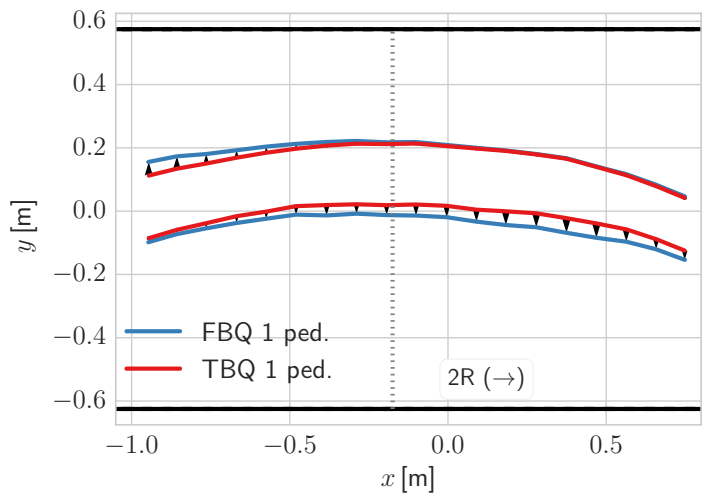

(b)

Figure 6 Preferred position bands for single pedestrians (cf. (F1) in Sec. 3.2), frame- vs. trajectorybased query results (cf. App. A for the definition of the position bands). In opposition to Fig. 5, and for the sake of readability, only the upper and the lower limit (in $y$ ) of the bands are retained. Pedestrians walking right-to-left and left-to-right are reported respectively in panel (a) and (b). Preferred position bands are in red for trajectory-based queries and in blue for frame-based queries. The black arrows indicate the displacement from the trajectory- to the frame-based cases. The black vertical dotted lines indicate the longitudinal center of the landing. (Measurement counts in Tab. 1 for FBQ and in Tab. 2 for TBQ).

In Fig. 6 we report the bands of preferred positions from frame- and trajectory-based queries of single pedestrians walking in either direction (i.e., number of people observed in the facility is one. See cases $(Q 1)$ and $(Q 3)$ in Sec. 2). Consistently with the subplots " $(1 \times \leftarrow, 0 \times \rightarrow)$ " and " $(0 \times \leftarrow, 1 \times \rightarrow)$ " in Fig. 5, the preferred position bands are almost exactly a translation of one another of about $20 \mathrm{~cm}$ in the $y$ direction. Although the relative position of the bands conforms with the cultural habit of keeping the driving side (cf. e.g. [30]), an influence of the landing geometry on the trajectories cannot be excluded. In fact, the shape of the facility limits the sight on the staircases, hence right-hand side 


\begin{tabular}{lrrrr}
\hline & \multicolumn{2}{c}{$2 \mathrm{~L}(\leftarrow)$} & \multicolumn{2}{c}{$2 \mathrm{R}(\rightarrow)$} \\
& frames & trajectories & frames & trajectories \\
\hline TBQ 1 ped. undist. & 801,476 & 32,791 & $1,099,129$ & 39,576 \\
TBQ 2 ped. counter-fl. & 81,588 & 2,339 & 91,545 & 2,339 \\
\hline
\end{tabular}

Table 2 Total number of frames and of unique trajectories selected by trajectory-based queries of undisturbed pedestrians and counter-flowing pairs (cf. (F1)-(F2) in Sec. 3.2). The TBQ of counterflowing pairs identifies 2,339 pairs of trajectories, each of which contributes with a possibly different number of frames as for each pair there are instants in which just one of the two pedestrians is present. Furthermore pedestrians going to the right walk typically slower (cf. Fig. 8(c,d)), thus the higher frame count (as the acquisition frequency is constant).

positions may help preventing collisions (cf. Fig. 10 and Fig. 13). We note that the bands from trajectory-based queries are symmetric with respect to the longitudinal axis of the corridor $(x \approx-0.1 \mathrm{~m})$, while this does not occur in the frame-based queries. In the framebased case, the entrance end of the bands expands more to the "upper" side of the corridor (larger $y$ values), and the exit end expands into the "lower" side of the corridor (smaller $y$ values).

The difference in the preferred position bands between the two queries comes from those pedestrians that, although alone in the frames selected by the FBQ, have met and/or will meet other pedestrians during their walk in the corridor, and these trajectories would be discarded by the TBQ. We systematically analyze such differences in Fig. 7(a,b), where we break up the considered FBQ separating data from the contributing scenarios, following the discussion in Sec. 2.1 and the expansion in Fig. 3. Frame-based queried data of single pedestrians contain contributions from

(A) the analogous TBQ (i.e. undisturbed motion with no interactions);

(B) co-flow (B1) and counter-flow (B2) scenarios including two pedestrians (i.e. interactions between exactly two peers);

(C) scenarios with three or more pedestrians (regardless of the appearance order/interactions).

Considering the weight of each scenario reported as the trajectory count in Fig. 7(c,d), we notice that case (A) is the prominent contributor in the query. In other words, whenever a pedestrian appears alone in our landing, most likely he or she walked undisturbed the whole time. This implies the tiny differences between the two query results in Fig. 6 . We notice also that the band enlargement between TBQ and FBQ, determined by cases (B-C), is mostly contributed by case (B1).

The walking velocity provides a second comparison environment for FBQ and TBQ of single pedestrian dynamics. Fig. 8 reports the average walking velocity at each space location within our recording window. Regardless of the query, the walking speed varies in space, showing contours that are roughly transversal to the curved walking paths. In the 


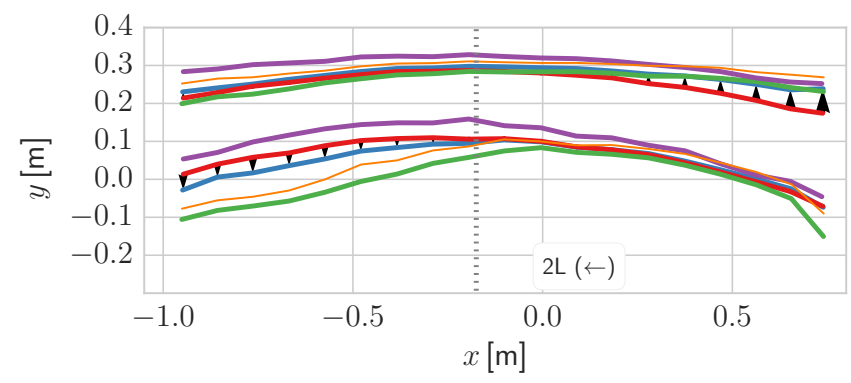

FBQ 1 p.

(A) TBQ 1 p. no int.

(B1) 2 p.int. co-flow

(B2) 2 p.int. counter-flow

(C) $>2$ p.int.

(a)

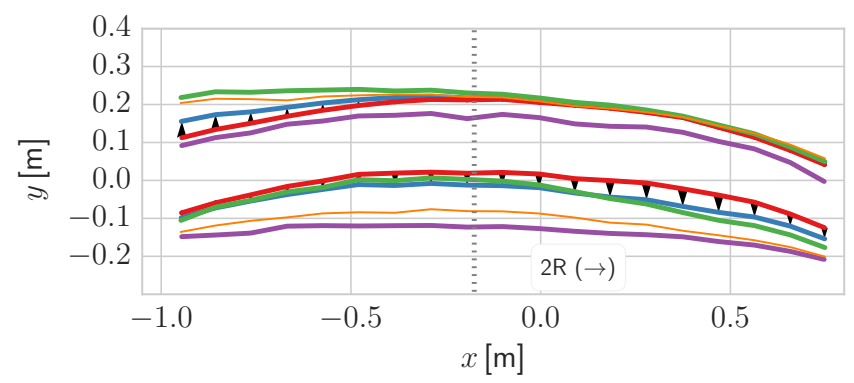

- FBQ $1 \mathrm{p}$

- (A) TBQ 1 p. no int.

- (B1) 2 p.int. co-flow

- (B2) 2 p.int. counter-flow

(C) $>2$ p.int.

(b)

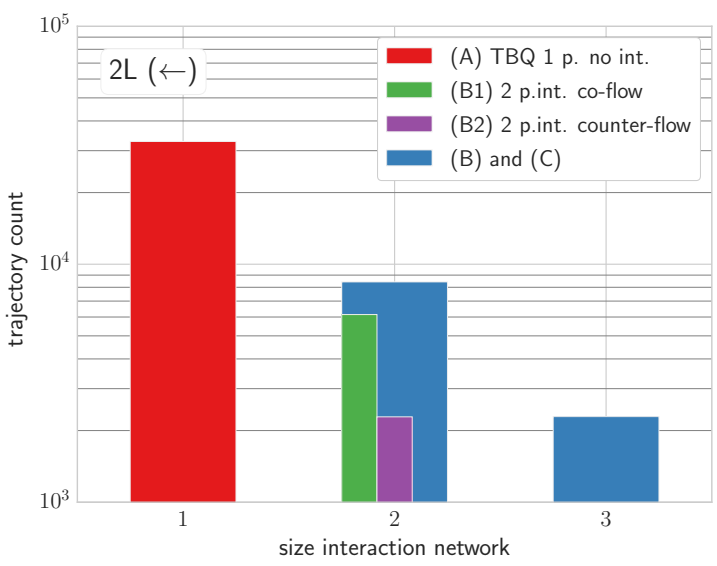

(c)

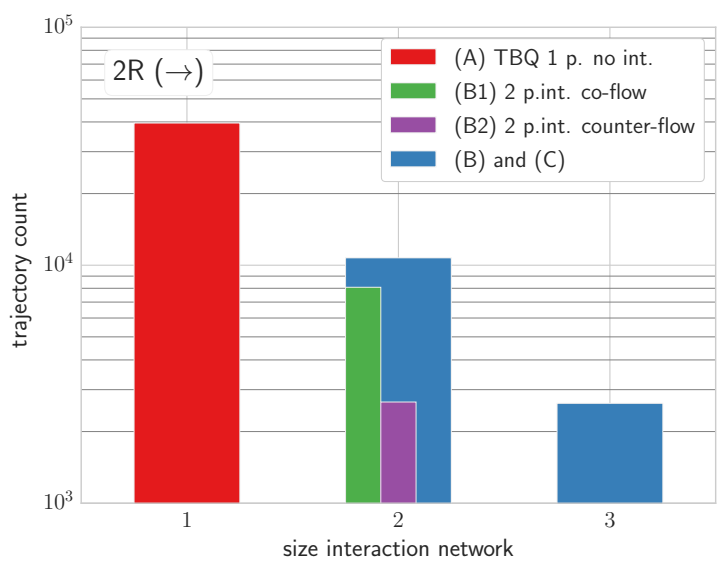

(d)

Figure 7 Contributions from different flow scenarios to the single pedestrian preferred position bands in Fig. 6. Panels (a,b) report the preferred position bands from FBQ (blue bounding lines) and TBQ of undisturbed pedestrians (red bounding lines, case (A) in Sec. 3.2), together with the specific contributions to the FBQ due exclusively to: (B1) pedestrians involved in a co-flow with another individual; (B2) counter-flow with another individual; (C) all the rest. The weight of each scenario, sorted by the size of the interaction network (horizontal axis, cf. Fig. 3), is reported in panels $(\mathrm{c}, \mathrm{d})$ in terms of the associated count of trajectories. As in Fig. 3 we truncate the plots at interaction network size equal to 3 . (For computing efficiency reasons the comparisons in these plots are evaluated considering 3,000,000 measurements out of 5,401,605). 


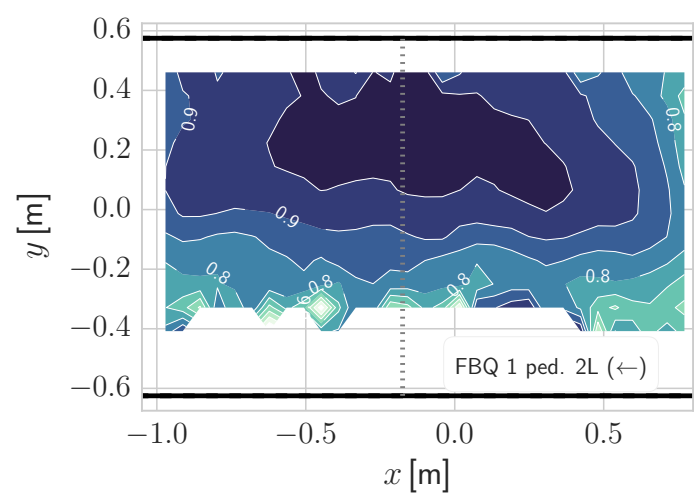

(a)

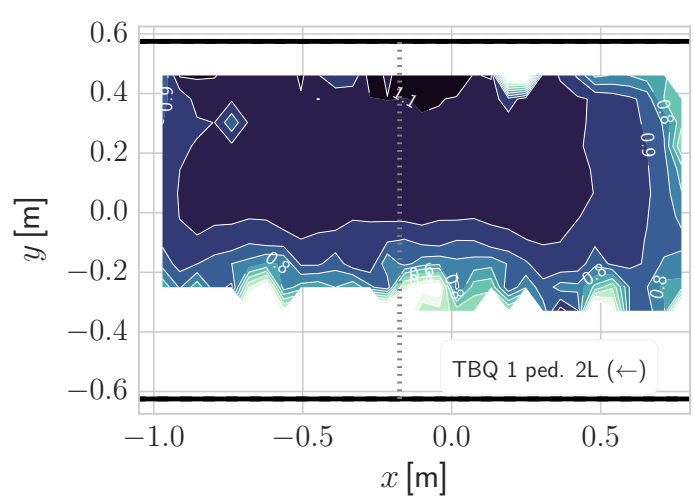

(c)

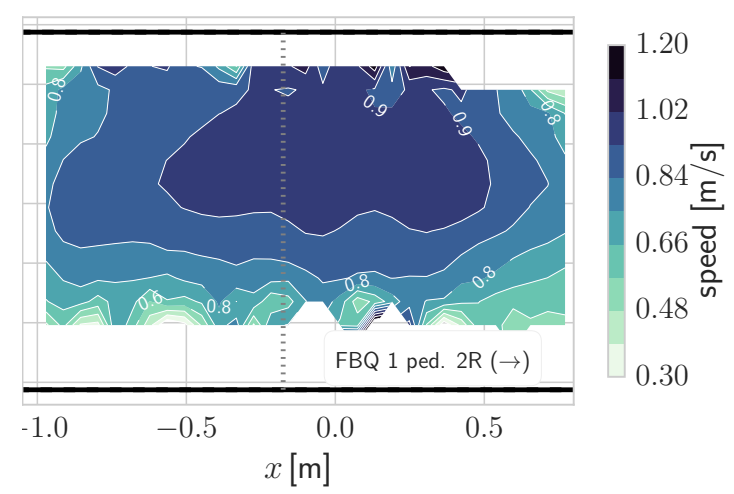

(b)

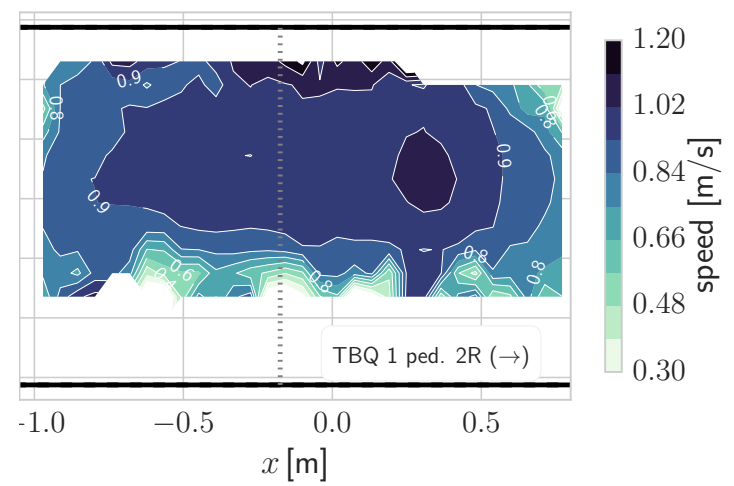

(d)

Figure 8 Average walking speed in space, selecting single pedestrian data via FBQ (a,b) and TBQ (c,d). Pedestrians walking right-to-left are in panels $(\mathrm{a}, \mathrm{c})$, while pedestrians walking left-to-right are in panels $(b, d)$. Walking velocities measured according to FBQ are lower than in the TBQ case due to mutual interactions.

first half of the corridor pedestrians arriving from the first staircase go through an acceleration phase, to decelerate in the second half reaching the second staircase. In both cases we observe a $30 \%$ speed variation. The acceleration region measured stretches longer in span-wise direction than the deceleration region. Furthermore, pedestrians going left-toright walk slower than pedestrians going right-to-left. This is consistent with the dynamics on the staircases themselves, in which, as intuition suggests, descending pedestrians move faster (e.g. [31]). Beside these similarities, we observe lower speed in FBQ results than in TBQ results, for both walking directions. Once more, in the FBQ data are included from pedestrians having a rich interaction network. Presence of further pedestrians (yet in frames not selected by FBQ) and the related reactions yield speed reductions. Data selected from TBQ are also significantly less symmetric around the longitudinal axis of the corridor.

Discrepancies between FBQ and TBQ become substantial when analyzing more complex dynamics scenarios, e.g. the counter-flowing pair case. In Fig. 9 we report a twofold 


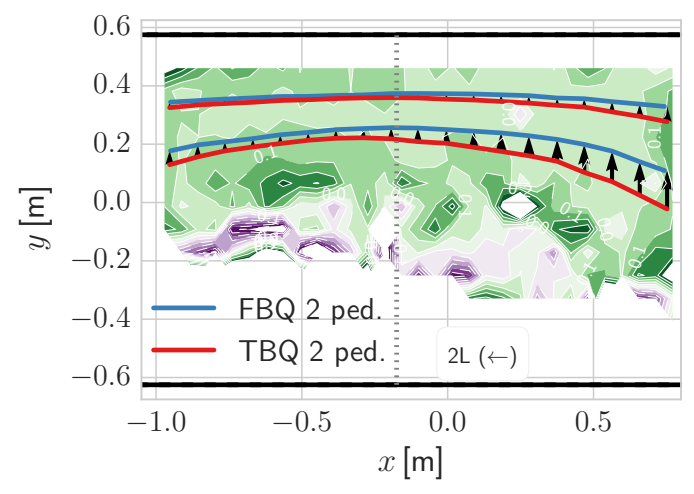

(a)

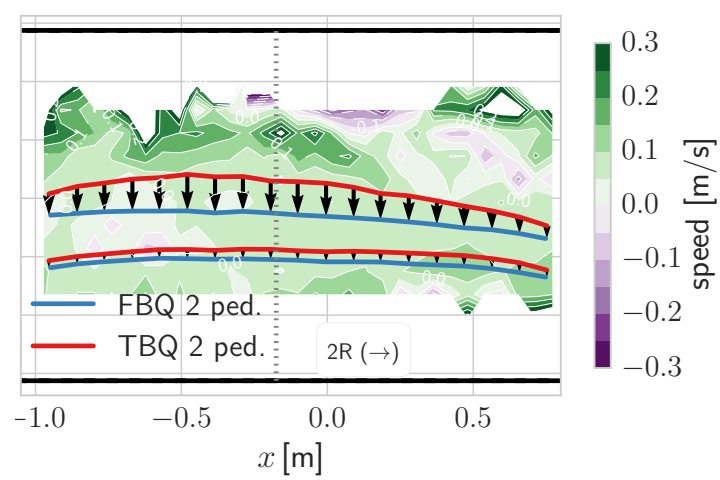

(b)

Figure 9 Comparison of FBQ and TBQ in the case of pair counter-flow dynamics (cf. (F2) Sec. 3.2) in terms of preferred position band and walking velocity difference. The blue and red solid lines report the boundaries of the preferred position bands from respectively FBQ and TBQ cases. The black arrows identifies the local displacement of the band, which is significantly larger than in the single pedestrian case ((F1) in Sec. 3.2, cf. Fig. 6). The underlying color maps reports the difference of the average speed velocities (as TBQ speed minus FBQ speed). The local average velocity from TBQ is higher almost everywhere. (Measurement counts in Tab. 2).

comparison of FBQ and TBQ including the preferred walking band (as in Fig. 7) and a point-wise difference of the local walking speed. Preferred walking bands as shown by FBQ are narrower and well separated. Furthermore their width remain mostly unchanged across the facility span. On the contrary, bands from TBQ are wider at the entrance side and constrict along the span. Higher walking speed are furthermore recorded in case of TBQ almost everywhere in the recordings. These differences follow the querying approaches, in fact:

(D1) FBQ includes data from pedestrians having possibly complex interaction networks. Such queries can hence include trajectories whose unselected frames are part e.g. of counter-flow scenarios with more than two pedestrians. In these conditions the formation of two neat walking lanes for the two directions is expected (cf. Fig. 5);

(D2) TBQ includes all frames involving the two considered pedestrians. These comprise the pre-avoidance maneuver and post-avoidance maneuvers frames (topic of Sec. 3.3). In the first case pedestrians entering might have not yet seen the other individual, and in the second case the other individual might have passed by already and dropped any influence, thus the larger size of the position band;

(D3) the aspects in (D2) impact also on the walking velocity that is on average higher as less interactions occur. 


\subsection{Trajectory-based analysis of pair-wise interactions}

In this section we analyze and compare the two diluted pedestrian dynamics cases introduced in Sec. 3.2 via trajectory-based queries. The two scenarios are likely the simplest as far as the dynamics is concerned: interactions, if any, occur just to avoid one other pedestrian that always walks in the opposite direction. Moreover, because of the geometric setting, they feature asymmetries. The analysis employs once more preferred position bands, velocity fields and also average acceleration fields. Looking at accelerations in the social-force modeling [34] perspective, we discuss the pair counter-flow avoidance in terms of "interaction forces".

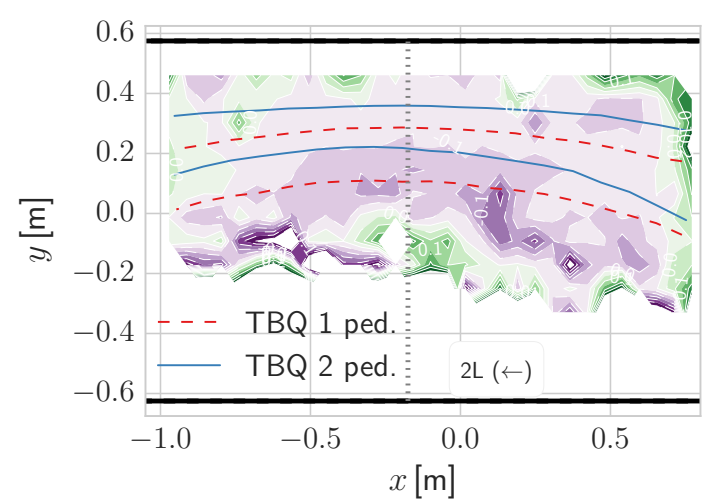

(a)

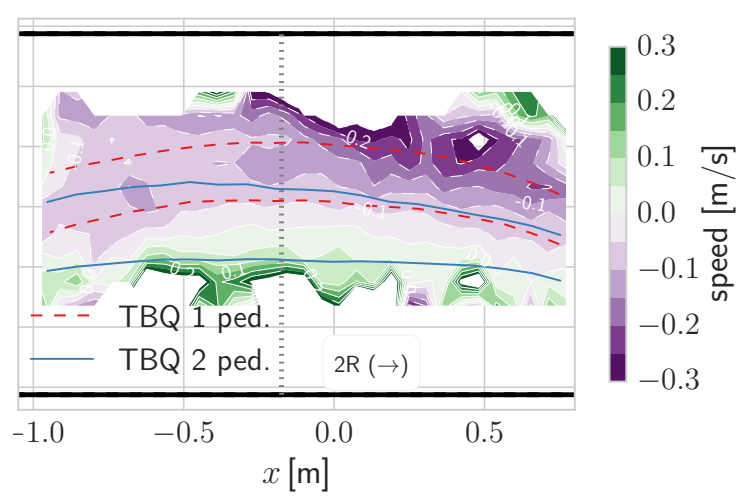

(b)

Figure 10 Comparison of undisturbed pedestrian and counter-flowing pair dynamics via TBQ. We report the boundaries of the preferred position bands in the undisturbed (dashed line) and pair (solid line) cases. The preferred position bands are shifted toward the relative right side of the corridor in the counter-flowing configuration due to collision avoidance. The underlying map shows the average velocity fields for the pair case minus average velocity field for undisturbed pedestrians. The difference is negative almost everywhere, as counter-flowing pedestrians slow down to ensure avoidance. Panels (a) and (b) report respectively the cases of pedestrians going right-to-left and left-to-right. (Measurement counts in Tab. 2).

Undisturbed pedestrians, conforming with all other flow conditions considered (Sec. 3.1 for FBQ), show an asymmetric dynamics with a side preference depending on the walking direction (see Fig. 6, TBQ cases). Furthermore, pedestrians going left-to-right exhibit higher walking velocities than in the right-to-left case (Fig. 8(c,d)). In both cases, the velocity field is non uniform in space. Direction-dependent differences increase in case of avoidance. Walking paths shift to the relative right to avoid collision, and the overlap between preferred position bands vanishes (see Fig. 10). Interestingly, the symmetry with respect to the landing longitudinal axis is lost. The displacement of the preferred position bands from the undisturbed pedestrian case to the counter-flowing pair case shows direction-related asymmetries too. Such displacement is on average ca. $40 \%$ larger in the left-to-right case. Furthermore, while for pedestrians going right-to-left the preferred position band shows an almost rigid displacement of $c a .10 \mathrm{~cm}$, in the left-to-right case the band deforms and its axis moves, on average, of $18 \mathrm{~cm}$. (cf. Fig. 10 and Fig. 11(a)). In 


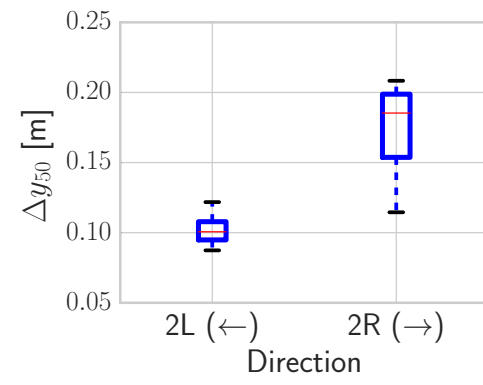

(a)

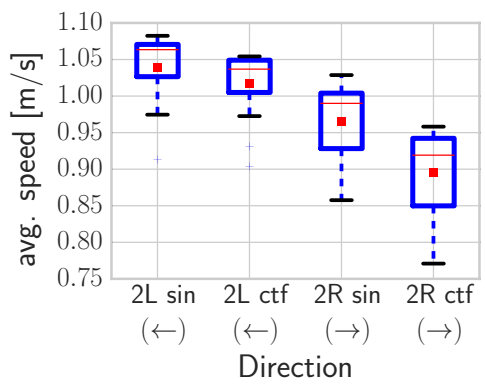

(b)

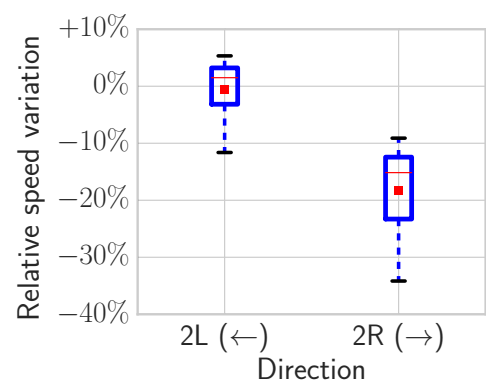

(c)

Figure 11 Quantitative comparisons between the single pedestrian undisturbed dynamics and the pair counter-flow dynamics in terms of position and velocity differences. (a) Displacement in absolute value of the layers of preferred positions from the undisturbed pedestrian case to the two pedestrians in counter-flow (cf. Fig. 10). We evaluate the transversal displacement of the median line of the path $y_{x, 50}$ (cf. explanation in App. A). We consider $\Delta y_{x, 50}^{2 L}=\left(y_{x, 50}^{\text {counter-flow, } 2 L}-\right.$ $\left.y_{x, 50}^{\text {undisturbed, } 2 L}\right)$ in the right-to-left case and $\Delta y_{x, 50}^{2 R}=-\left(y_{x, 50}^{\text {counter-flow, } 2 R}-y_{x, 50}^{\text {undisturbed,2R }}\right)$ in the left-to-right case. The box-plots report the distributions of $\Delta y_{x, 50}^{2 L}$ and of $\Delta y_{x, 50}^{2 R}$ across the landing span. The left-to-right pedestrians moved much more to the relative right when sharing the corridor with another pedestrian coming their way. (b) Comparison of walking speeds for undisturbed pedestrians and pair pedestrians in counter-flow. We evaluate the average speed at each span-wise location $x$, and for every direction we consider the relative difference of such velocity between undisturbed case ( $\sin$ ) and counter-flow (ctf). The distribution of the relative difference is reported by the box-plots. Descending (right-to-left) pedestrians walk faster than left-to-right pedestrian in both undisturbed and counter-flowing situations. Although slowing down occur in both counter-flowing directions, the effect is more pronounced for the left-toright pedestrians (cf. panel $\mathrm{c}$ and longitudinal deceleration in Fig. 13(b)). (c) Distribution of relative speed variation comparing undisturbed pedestrians and counter-flows of two. (a,b,c) The box-plots limit the first and third quartiles of the distributions, the whiskers identify the $5^{\text {th }}$ and $95^{\text {th }}$ percentiles. Red line reports the median and red dots the average values. (Measurement counts in Tab. 2).

comparison with undisturbed pedestrians, we observe an asymmetric drop in the walking speed: the left-to-right pedestrians have a velocity reduction of $18 \%$ on average, while the pedestrians walking right-to-left (and descending the stairs) show almost none. The speed reduction is more pronounced around the central horizontal axis $(y \approx 0 \mathrm{~m})$ where there is a higher chance of collision (cf. field in Fig. 10 and Fig. 11(b,c)). Consistently, higher walking speed are reached at the relative right hand side of the landing where collisions are unlikely.

These observations can be further discussed considering acceleration fields and interaction accelerations. Following a social force-like [34] approach, we model the acceleration of a pedestrian $\vec{a}_{p}$ as

$$
\vec{a}_{p}=\vec{a}_{p, d}+\vec{a}_{p, i}
$$

where $\vec{a}_{p, d}$ denotes the desired component of the acceleration, which in the modeling community is typically assigned a priori, and $\vec{a}_{p, i}$ is the perturbation to $\vec{a}_{p, d}$ for avoidance interaction. Generally $\vec{a}_{p, d}$ is modeled as a relaxation force toward a given desired veloc- 


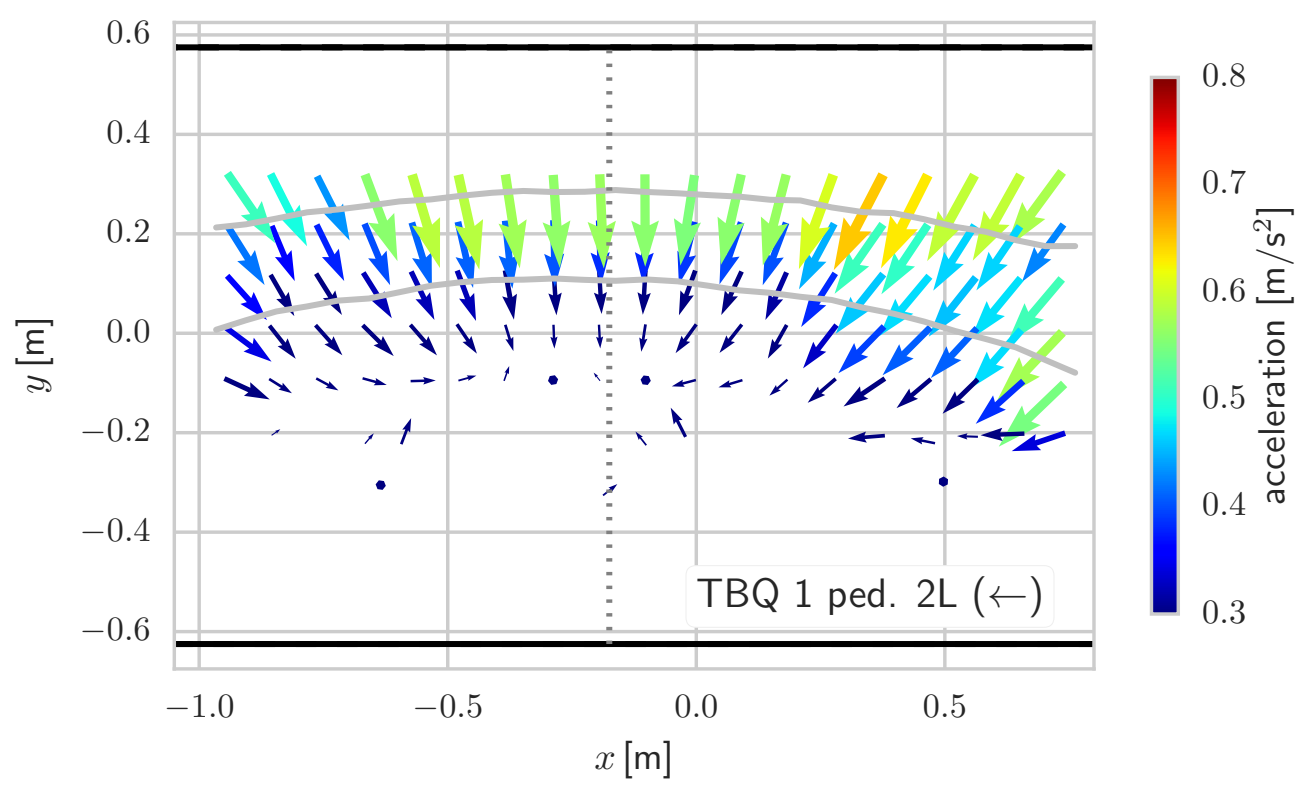

Figure 12 Average acceleration field for undisturbed pedestrians walking in right-to-left direction (cf. P2 in Fig. 2). The curved pedestrian motion following the U-shape of the corridor determines a centripetal acceleration. We measure an almost central acceleration field pointing to $c a$. $(x=-0.25, y=-0.10) \mathrm{m}$. The analogous acceleration field for pedestrians going left-to-right is omitted.(Measurement counts in Tab. 2).

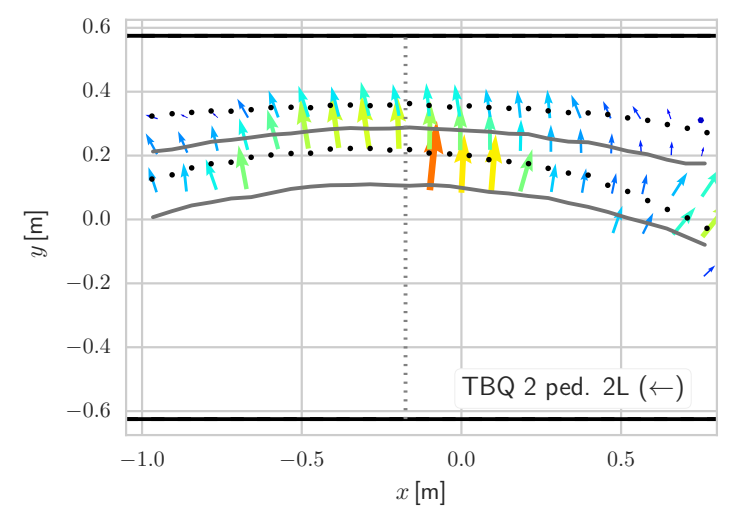

(a)

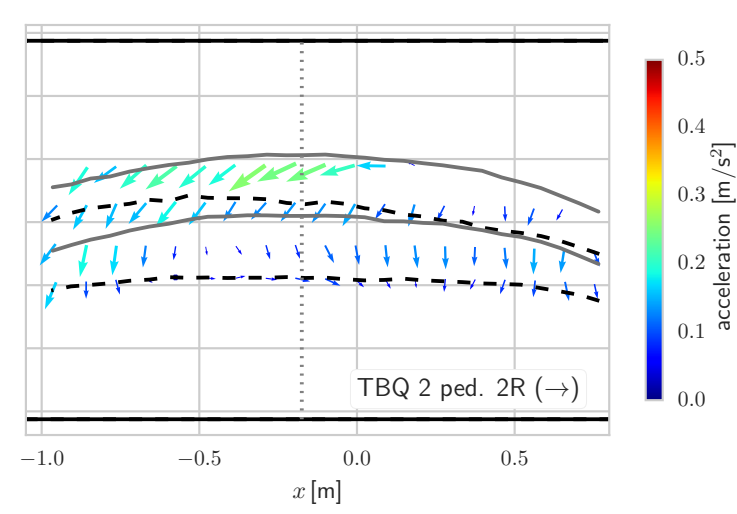

(b)

Figure 13 Average avoidance acceleration field (cf. Eq. 2) for two pedestrians counter-flowing (case P3-P4 in Fig. 2). Pedestrians walking in right-to-left and left-to-right directions are reported respectively in panels (a) and (b). In both cases the fields yield sidesteps on the relative right and a longitudinal speed reduction in the entering half of the landing. In the preferred walking band of undisturbed pedestrians (solid line) deceleration is stronger. The dashed line reports the preferred walking band for the two pedestrians case. (Measurement counts in Tab. 2). 
ity field. To analyze the counter-flow scenario, we assess the term $\vec{a}_{p, i}$. To this purpose, we assume that the acceleration of undisturbed pedestrians (from now on $\vec{a}_{p, 1}$ ) provides a good approximation, at least on average, to $\vec{a}_{p, d}$. In other words, we assume that undisturbed pedestrian move according to their desired acceleration. Notably, as in our setting pedestrians follow curved trajectories even when undisturbed, they constatly experience a centripetal acceleration (cf. Fig. 12 for an example of the average field). We extract the average interaction acceleration as

$$
\left\langle\vec{a}_{p, i}\right\rangle=\left\langle\vec{a}_{p}\right\rangle-\left\langle\vec{a}_{p, d}\right\rangle \approx\left\langle\vec{a}_{p}\right\rangle-\left\langle\vec{a}_{p, 1}\right\rangle,
$$

where $\langle\vec{a}\rangle$ is a local spatial average of $\vec{a}$ (cf. App. A for technical details). We report the avoidance acceleration fields for pedestrians going right-to-left and left-to-right in Fig. 13.

As expected, we notice that in both right-to-left and left-to-right cases the interaction acceleration points toward the relative right yielding the observed relative right drift of trajectories i.e. the displacement of the preferred position bands (cf. position bands in Fig. 13). Furthermore, consistent with the asymmetric speed variations shown in Fig. 11(c), longitudinal decelerations are visible mostly in the left-to-right case, especially in proximity of the entering side (i.e. the left end of the landing) and around the axis $y \approx 0$.

\section{Discussion}

Long-time measurements of pedestrian dynamics in real-life usually show wide heterogeneities containing randomly alternating instances of diverse flow conditions. This aspect marks a strong difference with laboratory-based data acquisition campaigns, in which flow conditions can be precisely defined and changed by the experimenter. In this perspective, before any further data analysis, a preparatory data selection step is mandatory in order to isolate, consistently with parameters expected to drive the flow (i.e. control parameters) occurrences of similar dynamics. Accidental aggregation of data from different flow conditions may yield biased statistical measurements potentially compromising the forthcoming physical analyses. Often this selection step has been (completely or partially) aided by visual assessments. This is a strong limiting factor when aiming at statistical dynamics analyses, which demand for extensive databases. To the best of our knowledge, automatizing this data selection step (query) defines an open challenge, for which we here suggest a tackling strategy. The current effort focuses and analyses the asymmetric pedestrian dynamics establishing in staircases landings. For this, we consider two possible querying schemas providing automatic aggregation of measurements based on expected control parameters and operating either at the frame level or at the trajectory level. Frame-based queries ensure the satisfaction of control parameters frame-by-frame, i.e. locally in time, possibly extracting measurements from parts of trajectories. On the opposite, trajectory-based queries ensure that given flow parameters are maintained throughout an entire trajectory before retaining it. This reflects the idea that, at least in a limited domain as the landing analyzed, pedestrian maintain a consistent, non-local in time and memory-based behavior. In fact, the dynamics of a pedestrian that in one 
time instant appears walking alone might have been perturbed by previous interactions. In this sense, for instance, a pedestrian cannot be said to feature an "undisturbed" motion unless he or she walked alone through the entire facility. To represent and perform trajectory-based queries we suggest a graph-based method. With one pass of the measurement database we build such graph in which interacting pedestrians are connected within connected components, here named interaction networks. Queries are abstracted as well in terms of graphs: each query agglomerates data from interaction networks with the same connectivity. Undisturbed pedestrians are for instance isolated, unconnected, nodes. Interacting pairs are connected components with two nodes and so on.

We employed the two query schemas to select automatically data for the investigation of our measurements database, obtained in a year-long experimental campaign. Specifically, we commented on the pedestrian dynamics depending on flow conditions, here identified through two control parameters: the number of pedestrians and their walking directions. As shown in Sec. 3.2, the employment of either query schema can make a significant difference in the data selected and thus in the following analysis. Frame-based queries have a simple definition and implementation, however they likely produce spurious data: e.g. the single pedestrians queries contain data from trajectories perturbed by many peers giving different velocity records. Similarly, the counter-flowing pairs case shows heavily different preferred position bands that, in the case of frame-based queries, are more confined as it would follow from many interactions (cf. Fig. 5).

In general, the U-shape of the landing combines with functional differences of walking directions (pedestrians walking to the left are going to a lower level in the building, the opposite happens for pedestrians going to the right) and yields asymmetries at the levels of preferred positions, velocities and also avoidance accelerations (social interaction forces). The quantitative differences found include, beside higher velocities for pedestrians globally descending, larger influences to the walking patterns of ascending pedestrians in case of a counter-flow. Further, we observed a strong walking side preference towards the driving side. Although it looks reasonable to expect that this asymmetry holds on any staircase landing, we cannot exclude an influence by the shape of the facility. In principle, ascending pedestrians, typically walking in the inner side, may have a different sight range on the environment than individuals descending, yielding lower speed.

A couple of further remarks should be noted from our definition of an interaction network. We introduce connection edges in the pedestrian relation graph after simultaneous appearance of individuals in our observation window. When employing trajectory-based queries we assume that interactions among pedestrians are limited to connected components of the graphs, i.e. to pedestrians' interaction networks. In principle, interactions might happen outside our recording windows and still play a role in the observed dynamics. Although this aspect is hard to assess, we expect that because of the limited extension of the landing it played negligible effects. In other words, we assume that if two pedestrians interact then, at a point, both appeared altogether in our observation window. Moreover, the spatial scale of our geometry is certainly relevant. Although it is reasonable to assume that two pedestrians appearing together in our observation window play a reciprocal influence on their dynamics, the same would not hold for larger geometrical settings (e.g. spanning beyond typical interaction ranges). In this cases, the sole 
simultaneous appearance does not justify the subsistence of an interaction. Generalizing the graph structure including geometric distances might help in treating such cases.

\section{A. Preferred position bands, speed and acceleration fields}

We evaluate preferred position bands, speed and acceleration fields via a spatial binning of the measurements from homogeneous sets obtained by either frame-based or trajectorybased queries (cf. Sec. 2). Given a homogeneous set of query results every detection $d$ has the form

$$
d=\left(t, p, x, y, u, w, a_{x}, a_{y}\right)
$$

where $t$ is the detection time, $p$ is an unique index for the detected pedestrian, $(x, y)$ is the position of the pedestrian at time $t, \vec{v}=(u, w)$ his/her velocity, $\vec{a}=\left(a_{x}, a_{y}\right)$ his/her acceleration.

To evaluate the preferred position band we extend the approach suggested in [12]. We bin the detection set $\{d\}$ with respect to the longitudinal position $x$ between $x=-1 \mathrm{~m}$ and $x=0.8 \mathrm{~m}$ in 40 equal bins. For each bin we consider the distribution of transversal positions $y_{x}$ (where the $x$ subscript indicates the dependence on the bin), and we take the $15^{\text {th }}$ and $85^{\text {th }}$ percentiles (indicated by $y_{x, 15}$ and $y_{x, 85}$ ) of the distribution to define the preferred position band.

We evaluate the displacement of the preferred position bands in Fig. 11(a) by considering in each bin the $50^{\text {th }}$ percentiles of $y_{x}: y_{x, 50}$. We compute the difference between the value for pedestrians undisturbed $\left(y_{x, 50}^{\text {undisturbed, } 2 L}\right.$, for the right-to-left case) and walking with one other individual in opposite direction $\left(y_{x, 50}^{\text {counter-flow }, 2 L}\right)$. We consider the distribution of the quantity obtained $\left(\Delta y_{x, 50}^{2 L}=\left(y_{x, 50}^{\text {counter-flow }, 2 L}-y_{x, 50}^{\text {undisturbed, } 2 L}\right)\right)$.

Velocity and acceleration fields are defined after a binning with respect to $x$ and $y$. For each bin we take respectively the average speed $\left\langle\sqrt{u^{2}+v^{2}}\right\rangle$ and the average acceleration $\langle\vec{a}\rangle=\left(\left\langle a_{x}\right\rangle,\left\langle a_{y}\right\rangle\right)$. For the velocity fields we employed 32 bins in $x$ direction within $[-1.0,0.8] \mathrm{m}$ and 20 bins in $y$ direction within $[-1.0,0.5] \mathrm{m}$. For the acceleration fields we employed 16 bins in $x$ direction within $[-1.0,0.8] \mathrm{m}$ and 7 bins in $y$ direction within $[-0.4,0.4] \mathrm{m}$.

Acknowledgements We thank A. Holten and G. Oerlemans (Eindhoven, NL) for their help in the establishment of the measurement setup at Eindhoven University of Technology and A. Liberzon (Tel Aviv, IL) for his help in the adaptation of the OpenPTV library. We acknowledge the support from the Brilliant Streets research program of the Intelligent Lighting Institute at the Eindhoven University of Technology, NL. This work is part of the JSTP research programme "Vision driven visitor behaviour analysis and crowd management" with project number 341-10-001, which is financed by the Netherlands Organisation for Scientific Research (NWO). Support from COST Action MP1305 "Flowing Matter" is also kindly acknowledged. 


\section{References}

[1] Corbetta, A., Lee, C., Muntean, A., Toschi, F.: Asymmetric pedestrian dynamics on a staircase landing from continuous measurements. In: Daamen, W., Knoop, V. (eds.) Traffic and Granular Flows '15, chap. 7. Springer (2016). doi:10.1007/978-3-319-33482-0_7

[2] Karamouzas, I., Skinner, B., Guy, S.J.: Universal power law governing pedestrian interactions. Phys. Rev. Lett. 113, 238701 (2014). doi:10.1103/PhysRevLett.113.238701

[3] Johansson, A., Helbing, D.: Analysis of empirical trajectory data of pedestrians. In: Pedestrian and Evacuation Dynamics 2008, pp. 203-214. Springer (2010). doi:10.1007/978-3-642-04504-2_15

[4] Helbing, D., Mukerji, P.: Crowd disasters as systemic failures: analysis of the love parade disaster. EPJ Data Science 1(1), 1-40 (2012). doi: 10.1140 / ep jds 7

[5] Johansson, A., Helbing, D., Shukla, P.: Specification of the social force pedestrian model by evolutionary adjustment to video tracking data. Adv. Complex Syst. 10(supp02), 271-288 (2007). doi:10.1142/s 0219525907001355

[6] Helbing, D., Johansson, A., Al-Abideen, H.Z.: Dynamics of crowd disasters: An empirical study. Phys. Rev. E 75(4), 046109 (2007). doi:10.1103/PhysRevE.75.046109

[7] Zhang, J., Seyfried, A.: Comparison of intersecting pedestrian flows based on experiments. Physica A 405, 316-325 (2014). doi:10.1016/j.physa.2014.03.004

[8] Brščić, D., Zanlungo, F., Kanda, T.: Density and velocity patterns during one year of pedestrian tracking. Transportation Research Procedia 2, 77-86 (2014). doi:10.1016/j.trpro.2014.09.011

[9] Mehner, W., Boltes, M., Mathias, M., Leibe, B.: Robust marker-based tracking for measuring crowd dynamics. In: Computer Vision Systems, pp. 445-455. Springer (2015). doi:10.1007/978-3-319-20904-3_40

[10] Boltes, M., Seyfried, A.: Collecting pedestrian trajectories. Neurocomputing 100, 127-133 (2013). doi:10.1016/j.neucom.2012.01.036

[11] Zhang, J., Klingsch, W., Schadschneider, A., Seyfried, A.: Transitions in pedestrian fundamental diagrams of straight corridors and T-junctions. J. Stat. Mech.-Theory E. 2011(06), P06004 (2011). doi:10.1088/1742-5468/2011/06/P 06004

[12] Corbetta, A., Bruno, L., Muntean, A., Toschi, F.: High statistics measurements of pedestrian dynamics. Transportation Research Procedia 2, 96-104 (2014). doi:10.1016/j.trpro.2014.09.013 
[13] Seer, S., Brändle, N., Ratti, C.: Kinects and human kinetics: A new approach for studying pedestrian behavior. Transport. Res. C-Emer. 48, 212-228 (2014). doi:10.1016/j.trc.2014.08.012

[14] Schadschneider, A., Klingsch, W., Klüpfel, H., Kretz, T., Rogsch, C., Seyfried, A.: Evacuation dynamics: Empirical results, modeling and applications. In: Encyclopedia of Complexity and Systems Science, pp. 3142-3176. Springer (2009). doi:10.1007/978-0-387-30440-3_187

[15] Roggen, D., Wirz, M., Tröster, G., Helbing, D.: Recognition of crowd behavior from mobile sensors with pattern analysis and graph clustering methods. Netw. Heterog. Media 6(3), 521-544 (2011). doi:10.3934/nhm.2011.6.521

[16] Corbetta, A.: Multiscale crowd dynamics: physical analysis, modeling and applications. Ph.D. thesis, Technische Universiteit Eindhoven (2016)

[17] Corbetta, A., Lee, C., Benzi, R., Muntean, A., Toschi, F.: Fluctuations around mean walking behaviours in diluted pedestrian flows. Phys. Rev. E, in press, arXiv preprint arXiv:1610.07429 [physics.soc-ph] (2017)

[18] Dalal, N., Triggs, B.: Histograms of oriented gradients for human detection. In: Computer Vision and Pattern Recognition, 2005. CVPR 2005. IEEE Computer Society Conference on, vol. 1, pp. 886-893. IEEE (2005). doi:10.1109/CVPR.2005.177

[19] Cao, S., Zhang, J., Salden, D., Ma, J., Shi, C., Zhang, R.: Pedestrian dynamics in single-file movement of crowd with different age compositions. Phys. Rev. E 94, 012312 (2016). doi: 10.1103 /PhysRevE.94.012312

[20] Garcimartín, A., Parisi, D., Pastor, J., Martín-Gómez, C., Zuriguel, I.: Flow of pedestrians through narrow doors with different competitiveness. J. Stat. Mech.-Theory E. 2016(4), 043402 (2016). doi:10.1088/1742-5468/2016/04/043402

[21] Corbetta, A., Meeusen, J., Lee, C., Toschi, F.: Continuous measurements of real-life bidirectional pedestrian flows on a wide walkway. In: Pedestrian and Evacuation Dynamics 2016, pp. 18-24. University of Science and Technology of China press (2016)

[22] Zanlungo, F., Brščić, D., Kanda, T.: Pedestrian group behaviour analysis under different density conditions. Transportation Research Procedia 2, 149-158 (2014). doi:10.1016/j.trpro.2014.09.020

[23] Zanlungo, F., Ikeda, T., Kanda, T.: Potential for the dynamics of pedestrians in a socially interacting group. Phys. Rev. E 89, 012811 (2014). doi:10.1103/PhysRevE.89.012811 
[24] Tamura, Y., Terada, Y., Yamashita, A., Asama, H.: Modelling behaviour patterns of pedestrians for mobile robot trajectory generation. Int. J. Adv. Robot. Syst. 10 (2013). doi:10.5772/56668

[25] Seitz, M., Seer, S., Klettner, S., Handel, O., Köster, G.: How do we wait? fundamentals, characteristics, and modeling implications. In: Daamen, W., Knoop, V. (eds.) Traffic and Granular Flows '15. Springer (2016)

[26] Hoskins, B., Milke, J.: Study of Movement Speeds Down Stairs. SpringerBriefs in Fire. Springer New York (2012). URL https: / / books.google.nl/books? $i d=J b 27 \circ M M f C i w C$

[27] Hoskins, B., Milke, J.: Differences in measurement methods for travel distance and area for estimates of occupant speed on stairs. Fire Safety J. 48, 49-57 (2012). doi:10.1016/j.firesaf.2011.12.009

[28] Ronchi, E., Reneke, P., Kuligowski, E., Peacock, R.: An analysis of evacuation travel paths on stair landings by means of conditional probabilities. Fire Safety J. 65, 30-40 (2014). doi:10.1016/j.firesaf.2014.02.001

[29] Peacock, R., Reneke, P., Kuligowski, E., Hagwood, C.: Movement on stairs during building evacuations. Fire Technol. pp. 1-27. doi:10.1007/s10694-016-0603-5

[30] Moussaïd, M., Helbing, D., Garnier, S., Johansson, A., Combe, M., Theraulaz, G.: Experimental study of the behavioural mechanisms underlying selforganization in human crowds. Proc. R. Soc. B (276), 2755-2762 (2009). doi:10.1098/rspb.2009.0405

[31] Fujiyama, T., Tyler, N.: Predicting the walking speed of pedestrians on stairs. Transport. Plan. Tech. 33(2), 177-202 (2010)

[32] Bondy, J., Murty, U.: Graph theory with applications, vol. 290. Macmillan London (1976)

[33] Hagberg, A., Schult, D., Swart, P.: Exploring network structure, dynamics, and function using NetworkX. In: Proceedings of the 7th Python in Science Conference (SciPy2008), pp. 11-15. Pasadena, CA USA (2008)

[34] Helbing, D., Molnár, P.: Social force model for pedestrian dynamics. Phys. Rev. E 51(5), 4282-4286 (1995). doi:10.1103/PhysRevE.51.4282

[35] Stanley, R.: Enumerative Combinatorics. Volume 2. Cambridge University Press (2001)

[36] Corbetta, A., Muntean, A., Vafayi, K.: Parameter estimation of social forces in pedestrian dynamics models via a probabilistic method. Math. Biosci. Eng. 12(2), 337-356 (2015). doi:10.3934/mbe.2015.12.337 
[37] Microsoft Corp.: Kinect for Xbox 360 (2011). URL http : / /www • xbox . com/ en-us/kinect/. Redmond, WA, USA.

[38] The OpenPTV Consortium: OpenPTV: Open source particle tracking velocimetry (since 2012). URL http: / / www . openptv . net /

[39] Willneff, J.: A spatio-temporal matching algorithm for $3 \mathrm{~d}$ particle tracking velocimetry. Ph.D. thesis, ETH Zürich (2003) 